\title{
Some common tripled fixed point results in two quasi-partial metric spaces
}

Feng $\mathrm{Gu}^{*}$

\author{
*Correspondence: \\ gufeng99@sohu.com \\ Institute of Applied Mathematics \\ and Department of Mathematics, \\ Hangzhou Normal University, \\ Hangzhou, Zhejiang 310036, China
}

Abstract

In this paper, we establish some new common tripled fixed point theorems for mappings defined on a set equipped with two quasi-partial metrics. We also provide illustrative examples in support of our new results. The results presented in this paper generalize the well-known comparable results in the literature due to Karapinar et al. [Math. Comput. Model. 57:2442-2448, 2013], and Shatanawi and Pitea [Fixed Point Theory Appl. 2013:153, 2013].

MSC: $47 \mathrm{H} 10 ; 54 \mathrm{H} 25$

Keywords: common tripled fixed point; tripled coincidence point; $w^{*}$-compatible mapping pairs; generalized metric space

\section{Introduction and preliminaries}

In 1994, Matthews [1] introduced the notion of partial metric spaces and extended the Banach contraction principle from metric spaces to partial metric spaces. Based on the notion of partial metric spaces, several authors (for example, [2-32]) obtained some fixed point results for mappings satisfying different contractive conditions. Very recently, Haghi et al. [33] showed in their interesting paper that some of fixed point theorems in partial metric spaces can be obtained from metric spaces.

In 2013, Karapinar et al. [34] introduced the concept of quasi-partial metric spaces and studied some fixed point problems on quasi-partial metric spaces.

The notion of partial metric space is given as follows.

Definition 1.1 (Matthews [1]) A partial metric on a nonempty set $X$ is a function $p: X \times$ $X \rightarrow \mathbb{R}^{+}$such that for all $x, y, z \in X$ :

(p1) $x=y \Leftrightarrow p(x, x)=p(x, y)=p(y, y)$,

(p2) $p(x, x) \leq p(x, y)$,

(p3) $p(x, y)=p(y, x)$

(p4) $p(x, y) \leq p(x, z)+p(z, y)-p(z, z)$.

A partial metric space is a pair $(X, p)$ such that $X$ is a nonempty set and $p$ is a partial metric on $X$.

Following Karapinar et al. [34], the notion of quasi-partial metric spaces is given as follows.

Definition 1.2 (Karapinar et al. [34]) A quasi-partial metric on nonempty set $X$ is a function $q: X \times X \rightarrow \mathbb{R}^{+}$which satisfies:

\section{照 Springer}

๑ $2014 \mathrm{Gu}$; licensee Springer. This is an Open Access article distributed under the terms of the Creative Commons Attribution License (http://creativecommons.org/licenses/by/2.0), which permits unrestricted use, distribution, and reproduction in any medium, provided the original work is properly cited. 
$\left(\mathrm{QPM}_{1}\right)$ If $q(x, x)=q(x, y)=q(y, y)$, then $x=y$,

$\left(\mathrm{QPM}_{2}\right) \quad q(x, x) \leq q(x, y)$,

$\left(\mathrm{QPM}_{3}\right) \quad q(x, x) \leq q(y, x)$, and

$\left(\mathrm{QPM}_{4}\right) q(x, y)+q(z, z) \leq q(x, z)+q(z, y)$ for all $x, y, z \in X$.

A quasi-partial metric space is a pair $(X, q)$ such that $X$ is a nonempty set and $q$ is a quasi-partial metric on $X$.

Let $q$ be a quasi-partial metric on set $X$. Then

$$
d_{q}(x, y)=q(x, y)+q(y, x)-q(x, x)-q(y, y)
$$

is a metric on $X$.

Definition 1.3 (Karapinar et al. [34]) Let $(X, q)$ be a quasi-partial metric space. Then we have the following.

(i) A sequence $\left\{x_{n}\right\}$ converges to a point $x \in X$ if and only if

$$
q(x, x)=\lim _{n \rightarrow \infty} q\left(x, x_{n}\right)=\lim _{n \rightarrow \infty} q\left(x_{n}, x\right)
$$

(ii) A sequence $\left\{x_{n}\right\}$ is called a Cauchy sequence if $\lim _{n, m \rightarrow \infty} q\left(x_{n}, x_{m}\right)$ and $\lim _{n, m \rightarrow \infty} q\left(x_{m}, x_{n}\right)$ exist (and are finite).

(iii) The quasi-partial metric space $(X, q)$ is said to be complete if every Cauchy sequence $\left\{x_{n}\right\}$ in $X$ converges, with respect to $\tau_{q}$, to a point $x \in X$ such that

$$
q(x, x)=\lim _{n, m \rightarrow \infty} q\left(x_{n}, x_{m}\right)=\lim _{n, m \rightarrow \infty} q\left(x_{n}, x_{m}\right) .
$$

Bhaskar and Lakshmikantham [35] introduced the concept of coupled fixed point and studied some nice coupled fixed point theorems. Later, Lakshmikantham and Ćirić [36] introduced the notion of a coupled coincidence point of mappings. For some works on a coupled fixed point, we refer the reader to [37-68].

For simplicity, we denote from now on $\underbrace{X \times X \times \cdots \times X}_{k \text { terms }}$ by $X^{k}$ where $k \in \mathbb{N}$ and $X$ is a nonempty set. We start by recalling some definitions.

Definition 1.4 (Bhaskar and Lakshmikantham [35]) An element $(x, y) \in X^{2}$ is called a coupled fixed point of the mapping $F: X^{2} \rightarrow X$ if $F(x, y)=x$ and $F(y, x)=y$.

Definition 1.5 (Lakshmikantham and Ćirić [36]) An element $(x, y) \in X^{2}$ is called

(i) a coupled coincidence point of the mappings $F: X^{2} \rightarrow X$ and $g: X \rightarrow X$ if $F(x, y)=g x$ and $F(y, x)=g y$, and $(g x, g y)$ is called a coupled point of coincidence;

(ii) a common coupled fixed point of mappings $F: X^{2} \rightarrow X$ and $g: X \rightarrow X$ if $F(x, y)=g x=x$ and $F(y, x)=g y=y$.

Definition 1.6 (Abbas et al. [37]) The mappings $F: X^{2} \rightarrow X$ and $g: X \rightarrow X$ are called $w$-compatible if $g F(x, y)=F(g x, g y)$ whenever $F(x, y)=g x$ and $F(y, x)=g y$.

In 2010, Samet and Vetro [38] introduced a fixed point of order $N \geq 3$. In particular, for $N=3$. we have the following definition. 
Definition 1.7 (Samet and Vetro [38]) An element $(x, y, z) \in X^{3}$ is called a tripled fixed point of a given mapping $F: X^{3} \rightarrow X$ if $F(x, y, z)=x, F(y, z, x)=y$, and $F(z, x, y)=z$.

Note that Berinde and Borcut [39] defined differently the notion of tripled fixed point in the case of ordered sets in order to keep true the mixed monotone property. For more details, see [39].

Definition 1.8 (Aydi et al. [40]) An element $(x, y, z) \in X^{3}$ is called

(i) a tripled coincidence point of mappings $F: X^{3} \rightarrow X$ and $g: X \rightarrow X$ if $F(x, y, z)=g x$, $F(y, z, x)=g y$, and $F(z, x, y)=g z$. In this case $(g x, g y, g z)$ is called a tripled point of coincidence;

(ii) a common tripled fixed point of mappings $F: X^{3} \rightarrow X$ and $g: X \rightarrow X$ if $F(x, y, z)=g x=x, F(y, z, x)=g y=y$, and $F(z, x, y)=g z=z$.

Definition 1.9 (Aydi et al. [40]) The mappings $F: X^{3} \rightarrow X$ and $g: X \rightarrow X$ are called $w$ compatible if $g F(x, y, z)=F(g x, g y, g z)$ whenever $F(x, y, z)=g x, F(y, z, x)=g y$, and $F(z, x, y)=$ $g z$.

Recently, Aydi and Abbas [41] obtained some tripled coincidence and fixed point results in partial metric space.

Very recently, Shatanawi and Pitea [42] obtained some common coupled fixed point results for a pair of mappings in quasi-partial metric space.

Theorem 1.1 (Shatanawi and Pitea [42]) Let $(X, q)$ be a quasi-partial metric space, $g$ : $X \rightarrow X$ and $F: X^{2} \rightarrow X$ be two mappings. Suppose that there exist $k_{1}, k_{2}$, and $k_{3}$ in $[0,1)$ with $k_{1}+k_{2}+k_{3}<1$ such that the condition

$$
\begin{aligned}
& q(F(x, y), F(u, v))+q(F(y, x), F(v, u)) \\
& \quad \leq k_{1}[q(g x, g u)+q(g y, g v)]+k_{2}[q(g x, F(x, y))+q(g y, F(y, x))] \\
& \quad+k_{3}[q(g u, F(u, v))+q(g v, F(v, u))]
\end{aligned}
$$

holds for all $x, y, u, v \in X$. Also, suppose we have the following hypotheses:

(i) $F(X \times X) \subset g(X)$.

(ii) $g(X)$ is a complete subspace of $X$ with respect to the quasi-partial metric $q$.

Then the mappings $F$ and $g$ have a coincidence point $(x, y)$ satisfying $g x=F(x, y)$ and $g y=F(y, x)$.

Moreover, if $F$ and $g$ are $w$-compatible, then $F$ and $g$ have a unique common coupled fixed point of the form $(x, x)$.

The aim of this article is to prove some new common tripled fixed point theorems for mappings defined on a set equipped with two quasi-partial metrics.

The following lemma is crucial in our work.

Lemma 1.1 (Shatanawi and Pitea [42]) Let $(X, q)$ be a quasi-partial metric space. Then the following statements hold true:

(i) If $q(x, y)=0$, then $x=y$.

(ii) If $x \neq y$, then $q(x, y)>0$ and $q(y, x)>0$. 
In this manuscript, we generalize, improve, enrich, and extend the above coupled common fixed point results. We also state some examples to illustrate our results. This paper can be considered as a continuation of the remarkable works of Karapinar et al. [34] and Shatanawi and Pitea [42].

\section{Main results}

Theorem 2.1 Let $q_{1}$ and $q_{2}$ be two quasi-partial metrics on $X$ such that $q_{2}(x, y) \leq q_{1}(x, y)$, for all $x, y \in X$, and $F: X^{3} \rightarrow X, g: X \rightarrow X$ be two mappings. Suppose that there exist $k_{1}$, $k_{2}, k_{3}, k_{4}$, and $k_{5}$ in $[0,1)$ with

$$
k_{1}+k_{2}+k_{3}+2 k_{4}+k_{5}<1
$$

such that the condition

$$
\begin{aligned}
& q_{1}(F(x, y, z), F(u, v, w))+q_{1}(F(y, z, x), F(v, w, u))+q_{1}(F(z, x, y), F(w, u, v)) \\
& \leq k_{1}\left[q_{2}(g x, g u)+q_{2}(g y, g v)+q_{2}(g z, g w)\right] \\
&+k_{2}\left[q_{2}(g x, F(x, y, z))+q_{2}(g y, F(y, z, x))+q_{2}(g z, F(z, x, y))\right] \\
&+k_{3}\left[q_{2}(g u, F(u, v, w))+q_{2}(g v, F(v, w, u))+q_{2}(g w, F(w, u, v))\right] \\
&+k_{4}\left[q_{2}(g x, F(u, v, w))+q_{2}(g y, F(v, w, u))+q_{2}(g z, F(w, u, v))\right] \\
&+k_{5}\left[q_{2}(g u, F(x, y, z))+q_{2}(g v, F(y, z, x))+q_{2}(g w, F(z, x, y))\right]
\end{aligned}
$$

holds for all $x, y, z, u, v, w \in X$. Also, suppose we have the following hypotheses:

(i) $F\left(X^{3}\right) \subset g(X)$.

(ii) $g(X)$ is a complete subspace of $X$ with respect to the quasi-partial metric $q_{1}$.

Then the mappings $F$ and $g$ have a tripled coincidence point $(x, y, z)$ satisfying

$$
g x=F(x, y, z)=g y=F(y, z, x)=g z=F(z, x, y) .
$$

Moreover, if $F$ and $g$ are $w$-compatible, then $F$ and $g$ have a unique common tripled fixed point of the form $(u, u, u)$.

Proof Let $x_{0}, y_{0}, z_{0} \in X$. Since $F\left(X^{3}\right) \subset g(X)$, we can choose $x_{1}, y_{1}, z_{1} \in X$ such that $g x_{1}=$ $F\left(x_{0}, y_{0}, z_{0}\right), g y_{1}=F\left(y_{0}, z_{0}, x_{0}\right)$ and $g z_{1}=F\left(z_{0}, x_{0}, y_{0}\right)$. Similarly, we can choose $x_{2}, y_{2}, z_{2} \in X$ such that $g x_{2}=F\left(x_{1}, y_{1}, z_{1}\right), g y_{2}=F\left(y_{1}, z_{1}, x_{1}\right)$, and $g z_{2}=F\left(z_{1}, x_{1}, y_{1}\right)$. Continuing in this way we construct three sequences $\left\{x_{n}\right\},\left\{y_{n}\right\}$, and $\left\{z_{n}\right\}$ in $X$ such that

$$
\begin{aligned}
& g x_{n+1}=F\left(x_{n}, y_{n}, z_{n}\right), \quad g y_{n+1}=F\left(y_{n}, z_{n}, x_{n}\right) \quad \text { and } \\
& g z_{n+1}=F\left(z_{n}, x_{n}, y_{n}\right), \quad \forall n \geq 0 .
\end{aligned}
$$

It follows from (2.2), (2.3), (QPM2), and (QMP4) that

$$
\begin{aligned}
& q_{1}\left(g x_{n}, g x_{n+1}\right)+q_{1}\left(g y_{n}, g y_{n+1}\right)+q_{1}\left(g z_{n}, g z_{n+1}\right) \\
& \quad=q_{1}\left(F\left(x_{n-1}, y_{n-1}, z_{n-1}\right), F\left(x_{n}, y_{n}, z_{n}\right)\right)+q_{1}\left(F\left(y_{n-1}, z_{n-1}, x_{n-1}\right), F\left(y_{n}, z_{n}, x_{n}\right)\right)
\end{aligned}
$$




$$
\begin{aligned}
& +q_{1}\left(F\left(z_{n-1}, x_{n-1}, y_{n-1}\right), F\left(z_{n}, x_{n}, y_{n}\right)\right) \\
& \leq k_{1}\left[q_{2}\left(g x_{n-1}, g x_{n}\right)+q_{2}\left(g y_{n-1}, g y_{n}\right)+q_{2}\left(g z_{n-1}, g z_{n}\right)\right] \\
& +k_{2}\left[q_{2}\left(g x_{n-1}, F\left(x_{n-1}, y_{n-1}, z_{n-1}\right)\right)+q_{2}\left(g y_{n-1}, F\left(y_{n-1}, z_{n-1}, x_{n-1}\right)\right)\right. \\
& \left.+q_{2}\left(g z_{n-1}, F\left(z_{n-1}, x_{n-1}, y_{n-1}\right)\right)\right] \\
& +k_{3}\left[q_{2}\left(g x_{n}, F\left(x_{n}, y_{n}, z_{n}\right)\right)+q_{2}\left(g y_{n}, F\left(y_{n}, z_{n}, x_{n}\right)\right)+q_{2}\left(g z_{n}, F\left(z_{n}, x_{n}, y_{n}\right)\right)\right] \\
& +k_{4}\left[q_{2}\left(g x_{n-1}, F\left(x_{n}, y_{n}, z_{n}\right)\right)+q_{2}\left(g y_{n-1}, F\left(y_{n}, z_{n}, x_{n}\right)\right)+q_{2}\left(g z_{n-1}, F\left(z_{n}, x_{n}, y_{n}\right)\right)\right] \\
& +k_{5}\left[q_{2}\left(g x_{n}, F\left(x_{n-1}, y_{n-1}, z_{n-1}\right)\right)+q_{2}\left(g y_{n}, F\left(y_{n-1}, z_{n-1}, x_{n-1}\right)\right)\right. \\
& \left.+q_{2}\left(g z_{n}, F\left(z_{n-1}, x_{n-1}, y_{n-1}\right)\right)\right] \\
& =\left(k_{1}+k_{2}\right)\left[q_{2}\left(g x_{n-1}, g x_{n}\right)+q_{2}\left(g y_{n-1}, g y_{n}\right)+q_{2}\left(g z_{n-1}, g z_{n}\right)\right] \\
& +k_{3}\left[q_{2}\left(g x_{n}, g x_{n+1}\right)+q_{2}\left(g y_{n}, g y_{n+1}\right)+q_{2}\left(g z_{n}, g z_{n+1}\right)\right] \\
& +k_{4}\left[q_{2}\left(g x_{n-1}, g x_{n+1}\right)+q_{2}\left(g y_{n-1}, g y_{n+1}\right)+q_{2}\left(g z_{n-1}, g z_{n+1}\right)\right] \\
& +k_{5}\left[q_{2}\left(g x_{n}, g x_{n}\right)+q_{2}\left(g y_{n}, g y_{n}\right)+q_{2}\left(g z_{n}, g z_{n}\right)\right] \\
& \leq\left(k_{1}+k_{2}\right)\left[q_{2}\left(g x_{n-1}, g x_{n}\right)+q_{2}\left(g y_{n-1}, g y_{n}\right)+q_{2}\left(g z_{n-1}, g z_{n}\right)\right] \\
& +k_{3}\left[q_{2}\left(g x_{n}, g x_{n+1}\right)+q_{2}\left(g y_{n}, g y_{n+1}\right)+q_{2}\left(g z_{n}, g z_{n+1}\right)\right] \\
& +k_{4}\left[q_{2}\left(g x_{n-1}, g x_{n}\right)+q_{2}\left(g x_{n}, g x_{n+1}\right)-q_{2}\left(g x_{n}, g x_{n}\right)\right. \\
& +q_{2}\left(g y_{n-1}, g y_{n}\right)+q_{2}\left(g y_{n}, g y_{n+1}\right)-q_{2}\left(g y_{n}, g y_{n}\right) \\
& \left.+q_{2}\left(g z_{n-1}, g z_{n}\right)+q_{2}\left(g z_{n}, g z_{n+1}\right)-q_{2}\left(g z_{n}, g z_{n}\right)\right] \\
& +k_{5}\left[q_{2}\left(g x_{n}, g x_{n+1}\right)+q_{2}\left(g y_{n}, g y_{n+1}\right)+q_{2}\left(g z_{n}, g z_{n+1}\right)\right] \\
& \leq\left(k_{1}+k_{2}+k_{4}\right)\left[q_{2}\left(g x_{n-1}, g x_{n}\right)+q_{2}\left(g y_{n-1}, g y_{n}\right)+q_{2}\left(g z_{n-1}, g z_{n}\right)\right] \\
& +\left(k_{3}+k_{4}+k_{5}\right)\left[q_{2}\left(g x_{n}, g x_{n+1}\right)+q_{2}\left(g y_{n}, g y_{n+1}\right)+q_{2}\left(g z_{n}, g z_{n+1}\right)\right] \\
& \leq\left(k_{1}+k_{2}+k_{4}\right)\left[q_{1}\left(g x_{n-1}, g x_{n}\right)+q_{1}\left(g y_{n-1}, g y_{n}\right)+q_{1}\left(g z_{n-1}, g z_{n}\right)\right] \\
& +\left(k_{3}+k_{4}+k_{5}\right)\left[q_{1}\left(g x_{n}, g x_{n+1}\right)+q_{1}\left(g y_{n}, g y_{n+1}\right)+q_{1}\left(g z_{n}, g z_{n+1}\right)\right],
\end{aligned}
$$

which implies that

$$
\begin{aligned}
& q_{1}\left(g x_{n}, g x_{n+1}\right)+q_{1}\left(g y_{n}, g y_{n+1}\right)+q_{1}\left(g z_{n}, g z_{n+1}\right) \\
& \quad \leq \frac{k_{1}+k_{2}+k_{4}}{1-k_{3}-k_{4}-k_{5}}\left[q_{1}\left(g x_{n-1}, g x_{n}\right)+q_{1}\left(g y_{n-1}, g y_{n}\right)+q_{1}\left(g z_{n-1}, g z_{n}\right)\right] .
\end{aligned}
$$

Put $k=\frac{k_{1}+k_{2}+k_{4}}{1-k_{3}-k_{4}-k_{5}}$. Obviously, $0 \leq k<1$. Repetition of the above inequality (2.4) $n$ times, we get

$$
\begin{aligned}
& q_{1}\left(g x_{n}, g x_{n+1}\right)+q_{1}\left(g y_{n}, g y_{n+1}\right)+q_{1}\left(g z_{n}, g z_{n+1}\right) \\
& \quad \leq k^{n}\left[q_{1}\left(g x_{0}, g x_{1}\right)+q_{1}\left(g y_{0}, g y_{1}\right)+q_{1}\left(g z_{0}, g z_{1}\right)\right] .
\end{aligned}
$$

Next, we shall prove that $\left\{g x_{n}\right\},\left\{g y_{n}\right\}$, and $\left\{g z_{n}\right\}$ are Cauchy sequences in $g(X)$. 
In fact, for each $n, m \in \mathbb{N}, m>n$, from (QPM4) and (2.5) we have

$$
\begin{aligned}
& q_{1}\left(g x_{n}, g x_{m}\right)+q_{1}\left(g y_{n}, g y_{m}\right)+q_{1}\left(g z_{n}, g z_{m}\right) \\
& \quad \leq \sum_{i=n}^{m-1}\left[q_{1}\left(g x_{i}, g x_{i+1}\right)+q_{1}\left(g y_{i}, g y_{i+1}\right)+q_{1}\left(g z_{i}, g z_{i+1}\right)\right] \\
& \quad \leq \sum_{i=n}^{m-1} k^{i}\left[q_{1}\left(g x_{0}, g x_{1}\right)+q_{1}\left(g y_{0}, g y_{1}\right)+q_{1}\left(g z_{0}, g z_{1}\right)\right] \\
& \quad \leq \frac{k^{n}}{1-k}\left[q_{1}\left(g x_{0}, g x_{1}\right)+q_{1}\left(g y_{0}, g y_{1}\right)+q_{1}\left(g z_{0}, g z_{1}\right)\right] .
\end{aligned}
$$

This implies that

$$
\lim _{n, m \rightarrow \infty}\left[q_{1}\left(g x_{n}, g x_{m}\right)+q_{1}\left(g y_{n}, g y_{m}\right)+q_{1}\left(g z_{n}, g z_{m}\right)\right]=0,
$$

and so

$$
\begin{aligned}
& \lim _{n, m \rightarrow \infty} q_{1}\left(g x_{n}, g x_{m}\right)=0, \quad \lim _{n, m \rightarrow \infty} q_{1}\left(g y_{n}, g y_{m}\right)=0 \quad \text { and } \\
& \lim _{n, m \rightarrow \infty} q_{1}\left(g z_{n}, g z_{m}\right)=0 .
\end{aligned}
$$

By similar arguments as above, we can show that

$$
\begin{aligned}
& \lim _{n, m \rightarrow \infty} q_{1}\left(g x_{m}, g x_{n}\right)=0, \quad \lim _{n, m \rightarrow \infty} q_{1}\left(g y_{m}, g y_{n}\right)=0 \quad \text { and } \\
& \lim _{n, m \rightarrow \infty} q_{1}\left(g z_{m}, g z_{n}\right)=0 .
\end{aligned}
$$

Hence $\left\{g x_{n}\right\},\left\{g y_{n}\right\}$, and $\left\{g z_{n}\right\}$ are Cauchy sequences in $\left(g X, q_{1}\right)$. Since $\left(g X, q_{1}\right)$ is complete, there exist $g x, g y, g z \in g(X)$ such that $\left\{g x_{n}\right\}$, $\left\{g y_{n}\right\}$, and $\left\{g z_{n}\right\}$ converge to $g x, g y$, and $g z$ with respect to $\tau_{q_{1}}$, that is,

$$
\begin{aligned}
q_{1}(g x, g x) & =\lim _{n \rightarrow \infty} q_{1}\left(g x, g x_{n}\right)=\lim _{n \rightarrow \infty} q_{1}\left(g x_{n}, g x\right) \\
& =\lim _{n, m \rightarrow \infty} q_{1}\left(g x_{m}, g x_{n}\right)=\lim _{n, m \rightarrow \infty} q_{1}\left(g x_{n}, g x_{m}\right), \\
q_{1}(g y, g y) & =\lim _{n \rightarrow \infty} q_{1}\left(g y, g y_{n}\right)=\lim _{n \rightarrow \infty} q_{1}\left(g y_{n}, g y\right) \\
& =\lim _{n, m \rightarrow \infty} q_{1}\left(g y_{m}, g y_{n}\right)=\lim _{n, m \rightarrow \infty} q_{1}\left(g y_{n}, g y_{m}\right),
\end{aligned}
$$

and

$$
\begin{aligned}
q_{1}(g z, g z) & =\lim _{n \rightarrow \infty} q_{1}\left(g z, g z_{n}\right)=\lim _{n \rightarrow \infty} q_{1}\left(g z_{n}, g z\right) \\
& =\lim _{n, m \rightarrow \infty} q_{1}\left(g z_{m}, g z_{n}\right)=\lim _{n, m \rightarrow \infty} q_{1}\left(g z_{n}, g z_{m}\right) .
\end{aligned}
$$


Combining (2.7)-(2.11), we have

$$
\begin{aligned}
q_{1}(g x, g x) & =\lim _{n \rightarrow \infty} q_{1}\left(g x, g x_{n}\right)=\lim _{n \rightarrow \infty} q_{1}\left(g x_{n}, g x\right) \\
& =\lim _{n, m \rightarrow \infty} q_{1}\left(g x_{m}, g x_{n}\right)=\lim _{n, m \rightarrow \infty} q_{1}\left(g x_{n}, g x_{m}\right)=0, \\
q_{1}(g y, g y) & =\lim _{n \rightarrow \infty} q_{1}\left(g y, g y_{n}\right)=\lim _{n \rightarrow \infty} q_{1}\left(g y_{n}, g y\right) \\
& =\lim _{n, m \rightarrow \infty} q_{1}\left(g y_{m}, g y_{n}\right)=\lim _{n, m \rightarrow \infty} q_{1}\left(g y_{n}, g y_{m}\right)=0,
\end{aligned}
$$

and

$$
\begin{aligned}
q_{1}(g z, g z) & =\lim _{n \rightarrow \infty} q_{1}\left(g z, g z_{n}\right)=\lim _{n \rightarrow \infty} q_{1}\left(g z_{n}, g z\right) \\
& =\lim _{n, m \rightarrow \infty} q_{1}\left(g z_{m}, g z_{n}\right)=\lim _{n, m \rightarrow \infty} q_{1}\left(g z_{n}, g z_{m}\right)=0 .
\end{aligned}
$$

On the other hand, by (QMP4) we obtain

$$
\begin{aligned}
q_{1}\left(g x_{n+1}, F(x, y, z)\right) & \leq q_{1}\left(g x_{n+1}, g x\right)+q_{1}(g x, F(x, y, z))-q_{1}(g x, g x) \\
& \leq q_{1}\left(g x_{n+1}, g x\right)+q_{1}(g x, F(x, y, z)) \\
& \leq q_{1}\left(g x_{n+1}, g x\right)+q_{1}\left(g x, g x_{n+1}\right)+q_{1}\left(g x_{n+1}, F(x, y, z)\right)-q_{1}\left(g x_{n+1}, g x_{n+1}\right) \\
& \leq q_{1}\left(g x_{n+1}, g x\right)+q_{1}\left(g x, g x_{n+1}\right)+q_{1}\left(g x_{n+1}, F(x, y, z)\right) .
\end{aligned}
$$

Letting $n \rightarrow \infty$ in the above inequalities and using (2.12), we have

$$
\lim _{n \rightarrow \infty} q_{1}\left(g x_{n+1}, F(x, y, z)\right) \leq q_{1}(g x, F(x, y, z)) \leq \lim _{n \rightarrow \infty} q_{1}\left(g x_{n+1}, F(x, y, z)\right) .
$$

That is,

$$
\lim _{n \rightarrow \infty} q_{1}\left(g x_{n+1}, F(x, y, z)\right)=q_{1}(g x, F(x, y, z)) .
$$

Similarly, we have

$$
\lim _{n \rightarrow \infty} q_{1}\left(g y_{n+1}, F(y, z, x)\right)=q_{1}(g y, F(y, z, x))
$$

and

$$
\lim _{n \rightarrow \infty} q_{1}\left(g y_{n+1}, F(z, x, y)\right)=q_{1}(g z, F(z, x, y)) .
$$

Now we prove that $F(x, y, z)=g x, F(y, z, x)=g y$, and $F(z, x, y)=g z$. In fact, it follows from (2.2) and (2.3) that

$$
\begin{aligned}
& q_{1}\left(g x_{n+1}, F(x, y, z)\right)+q_{1}\left(g y_{n+1}, F(y, z, x)\right)+q_{1}\left(g z_{n+1}, F(z, x, y)\right) \\
& \quad=q_{1}\left(F\left(x_{n}, y_{n}, z_{n}\right), F(x, y, z)\right)+q_{1}\left(F\left(y_{n}, z_{n}, x_{n}\right), F(y, z, x)\right)+q_{1}\left(F\left(z_{n}, x_{n}, y_{n}\right), F(z, x, y)\right) \\
& \quad \leq k_{1}\left[q_{2}\left(g x_{n}, g x\right)+q_{2}\left(g y_{n}, g y\right)+q_{2}\left(g z_{n}, g z\right)\right]
\end{aligned}
$$




$$
\begin{aligned}
+ & k_{2}\left[q_{2}\left(g x_{n}, F\left(x_{n}, y_{n}, z_{n}\right)\right)+q_{2}\left(g y_{n}, F\left(y_{n}, z_{n}, x_{n}\right)\right)+q_{2}\left(g z_{n}, F\left(z_{n}, x_{n}, y_{n}\right)\right)\right] \\
& +k_{3}\left[q_{2}(g x, F(x, y, z))+q_{2}(g y, F(y, z, x))+q_{2}(g z, F(z, x, y))\right] \\
& +k_{4}\left[q_{2}\left(g x_{n}, F(x, y, z)\right)+q_{2}\left(g y_{n}, F(y, z, x)\right)+q_{2}\left(g z_{n}, F(z, x, y)\right)\right] \\
+ & k_{5}\left[q_{2}\left(g x, F\left(x_{n}, y_{n}, z_{n}\right)\right)+q_{2}\left(g y, F\left(y_{n}, z_{n}, x_{n}\right)\right)+q_{2}\left(g z, F\left(z_{n}, x_{n}, y_{n}\right)\right)\right] \\
= & k_{1}\left[q_{2}\left(g x_{n}, g x\right)+q_{2}\left(g y_{n}, g y\right)+q_{2}\left(g z_{n}, g z\right)\right] \\
& +k_{2}\left[q_{2}\left(g x_{n}, g x_{n+1}\right)+q_{2}\left(g y_{n}, g y_{n+1}\right)+q_{2}\left(g z_{n}, g z_{n+1}\right)\right] \\
& +k_{3}\left[q_{2}(g x, F(x, y, z))+q_{2}(g y, F(y, z, x))+q_{2}(g z, F(z, x, y))\right] \\
& +k_{4}\left[q_{2}\left(g x_{n}, F(x, y, z)\right)+q_{2}\left(g y_{n}, F(y, z, x)\right)+q_{2}\left(g z_{n}, F(z, x, y)\right)\right] \\
& +k_{5}\left[q_{2}\left(g x, g x_{n+1}\right)+q_{2}\left(g y, g y_{n+1}\right)+q_{2}\left(g z, g z_{n+1}\right)\right] \\
\leq & k_{1}\left[q_{1}\left(g x_{n}, g x\right)+q_{1}\left(g y_{n}, g y\right)+q_{1}\left(g z_{n}, g z\right)\right] \\
+ & k_{2}\left[q_{1}\left(g x_{n}, g x_{n+1}\right)+q_{1}\left(g y_{n}, g y_{n+1}\right)+q_{1}\left(g z_{n}, g z_{n+1}\right)\right] \\
+ & k_{3}\left[q_{1}(g x, F(x, y, z))+q_{1}(g y, F(y, z, x))+q_{1}(g z, F(z, x, y))\right] \\
+ & k_{4}\left[q_{1}\left(g x_{n}, F(x, y, z)\right)+q_{1}\left(g y_{n}, F(y, z, x)\right)+q_{1}\left(g z_{n}, F(z, x, y)\right)\right] \\
+ & k_{5}\left[q_{1}\left(g x, g x_{n+1}\right)+q_{1}\left(g y, g y_{n+1}\right)+q_{1}\left(g z, g z_{n+1}\right)\right] .
\end{aligned}
$$

Letting $n \rightarrow \infty$ in the above inequality, using (2.12)-(2.17), we obtain

$$
\begin{aligned}
& q_{1}(g x, F(x, y, z))+q_{1}(g y, F(y, z, x))+q_{1}(g z, F(z, x, y)) \\
& \quad \leq\left(k_{3}+k_{4}\right)\left[q_{1}(g x, F(x, y, z))+q_{1}(g y, F(y, z, x))+q_{1}(g z, F(z, x, y))\right] .
\end{aligned}
$$

By (2.1) we have $k_{3}+k_{4}<1$. Hence, it follows from (2.18) that

$$
q_{1}(g x, F(x, y, z))+q_{1}(g y, F(y, z, x))+q_{1}(g z, F(z, x, y))=0 .
$$

This implies that

$$
q_{1}(g x, F(x, y, z))=q_{1}(g y, F(y, z, x))=q_{1}(g z, F(z, x, y))=0 .
$$

By Lemma 1.1, we get $F(x, y, z)=g x, F(y, z, x)=g y$, and $F(z, x, y)=g z$. Hence, $(g x, g y, g z)$ is a tripled point of coincidence of mappings $F$ and $g$.

Next, we will show that the tripled point of coincidence is unique. Suppose that $\left(x^{*}, y^{*}, z^{*}\right) \in X^{3}$ with $F\left(x^{*}, y^{*}, z^{*}\right)=g x^{*}, F\left(y^{*}, z^{*}, x^{*}\right)=g y^{*}$, and $F\left(z^{*}, x^{*}, y^{*}\right)=g z^{*}$. Using (2.2), (2.12), (2.13), (2.14), and $\left(\mathrm{QPM}_{3}\right)$, we obtain

$$
\begin{aligned}
& q_{1}\left(g x, g x^{*}\right)+q_{1}\left(g y, g y^{*}\right)+q_{1}\left(g z, g z^{*}\right) \\
&= q_{1}\left(F(x, y, z), F\left(x^{*}, y^{*}, z^{*}\right)\right)+q_{1}\left(F(y, z, x), F\left(y^{*}, z^{*}, x^{*}\right)\right)+q_{1}\left(F(z, x, y), F\left(z^{*}, x^{*}, y^{*}\right)\right) \\
& \leq k_{1}\left[q_{2}\left(g x, g x^{*}\right)+q_{2}\left(g y, g y^{*}\right)+q_{2}\left(z, z^{*}\right)\right] \\
&+k_{2}\left[q_{2}(g x, F(x, y, z))+q_{2}(g y, F(y, z, x))+q_{2}(g z, F(z, x, y))\right] \\
&+k_{3}\left[q_{2}\left(g x^{*}, F\left(x^{*}, y^{*}, z^{*}\right)\right)+q_{2}\left(g y^{*}, F\left(y^{*}, z^{*}, x^{*}\right)\right)+q_{2}\left(g z^{*}, F\left(z^{*}, x^{*}, y^{*}\right)\right)\right]
\end{aligned}
$$




$$
\begin{aligned}
& +k_{4}\left[q_{2}\left(g x, F\left(x^{*}, y^{*}, z^{*}\right)\right)+q_{2}\left(g y, F\left(y^{*}, z^{*}, x^{*}\right)\right)+q_{2}\left(g z^{*}, F\left(z^{*}, x^{*}, y^{*}\right)\right)\right] \\
& +k_{5}\left[q_{2}\left(g x^{*}, F(x, y, z)\right)+q_{2}\left(g y^{*}, F(y, z, x)\right)+q_{2}\left(g z^{*}, F(z, x, y)\right)\right] \\
= & k_{1}\left[q_{2}\left(g x, g x^{*}\right)+q_{2}\left(g y, g y^{*}\right)+q_{2}\left(g z, g z^{*}\right)\right] \\
& +k_{2}\left[q_{2}(g x, g x)+q_{2}(g y, g y)+q_{2}(g z, g z)\right] \\
& +k_{3}\left[q_{2}\left(g x^{*}, g x^{*}\right)+q_{2}\left(g y^{*}, g y^{*}\right)+q_{2}\left(g z^{*}, g z^{*}\right)\right] \\
& +k_{4}\left[q_{2}\left(g x, g x^{*}\right)+q_{2}\left(g y, g y^{*}\right)+q_{2}\left(g z, g z^{*}\right)\right] \\
& +k_{5}\left[q_{2}\left(g x^{*}, g x\right)+q_{2}\left(g y^{*}, g y\right)+q_{2}\left(g z^{*}, g z\right)\right] \\
\leq & \left(k_{1}+k_{4}\right)\left[q_{1}\left(g x, g x^{*}\right)+q_{1}\left(g y, g y^{*}\right)+q_{1}\left(g z, g z^{*}\right)\right] \\
& +k_{2}\left[q_{1}(g x, g x)+q_{1}(g y, g y)+q_{1}(g z, g z)\right] \\
& +k_{3}\left[q_{1}\left(g x^{*}, g x^{*}\right)+q_{1}\left(g y^{*}, g y^{*}\right)+q_{1}\left(g z^{*}, g z^{*}\right)\right] \\
& +k_{5}\left[q_{1}\left(g x^{*}, g x\right)+q_{1}\left(g y^{*}, g y\right)+q_{1}\left(g z^{*}, g z\right)\right] \\
\leq & \left(k_{1}+k_{3}+k_{4}\right)\left[q_{1}\left(g x, g x^{*}\right)+q_{1}\left(g y, g y^{*}\right)+q_{1}\left(g z, g z^{*}\right)\right] \\
& +k_{5}\left[q_{1}\left(g x^{*}, g x\right)+q_{1}\left(g y^{*}, g y\right)+q_{1}\left(g z^{*}, g z\right)\right] .
\end{aligned}
$$

This implies that

$$
\begin{aligned}
& q_{1}\left(g x, g x^{*}\right)+q_{1}\left(g y, g y^{*}\right)+q_{1}\left(g z, g z^{*}\right) \\
& \quad \leq \frac{k_{5}}{1-k_{1}-k_{3}-k_{4}} \cdot\left[q_{1}\left(g x^{*}, g x\right)+q_{1}\left(g y^{*}, g y\right)+q_{1}\left(g z^{*}, g z\right)\right] .
\end{aligned}
$$

Similarly, we have

$$
\begin{aligned}
& q_{1}\left(g x^{*}, g x\right)+q_{1}\left(g y^{*}, g y\right)+q_{1}\left(g z^{*}, g z\right) \\
& \quad \leq \frac{k_{5}}{1-k_{1}-k_{3}-k_{4}} \cdot\left[q_{1}\left(g x, g x^{*}\right)+q_{1}\left(g y, g y^{*}\right)+q_{1}\left(g z, g z^{*}\right)\right] .
\end{aligned}
$$

Substituting (2.20) into (2.19), we obtain

$$
\begin{aligned}
& q_{1}\left(g x, g x^{*}\right)+q_{1}\left(g y, g y^{*}\right)+q_{1}\left(g z, g z^{*}\right) \\
& \quad \leq\left(\frac{k_{5}}{1-k_{1}-k_{3}-k_{4}}\right)^{2} \cdot\left[q_{1}\left(g x, g x^{*}\right)+q_{1}\left(g y, g y^{*}\right)+q_{1}\left(g z, g z^{*}\right)\right] .
\end{aligned}
$$

Since $\frac{k_{5}}{1-k_{1}-k_{3}-k_{4}}<1$, from (2.21), we must have $q_{1}\left(g x, g x^{*}\right)=q_{1}\left(g y, g y^{*}\right)=q_{1}\left(g z, g z^{*}\right)=0$. By Lemma 1.1, we get $g x=g x^{*}, g y=g y^{*}$, and $g z=g z^{*}$, which implies that the uniqueness of the tripled point of coincidence of $F$ and $g$, that is, $(g x, g y, g z)$.

Next, we will show that $g x=g y=g z$. In fact, from (2.2), (2.12)-(2.14) we have

$$
\begin{aligned}
& q_{1}(g x, g y)+q_{1}(g y, g z)+q_{1}(g z, g x) \\
& \quad=q_{1}(F(x, y, z), F(y, z, x))+q_{1}(F(y, z, x), F(z, x, y))+q_{1}(F(z, x, y), F(x, y, z)) \\
& \quad \leq k_{1}\left[q_{2}(g x, g y)+q_{2}(g y, g z)+q_{2}(g z, g x)\right]
\end{aligned}
$$




$$
\begin{aligned}
+ & k_{2}\left[q_{2}(g x, F(x, y, z))+q_{2}(g y, F(y, z, x))+q_{2}(g z, F(z, x, y))\right] \\
+ & k_{3}\left[q_{2}(g y, F(y, z, x))+q_{2}(g z, F(z, x, y))+q_{2}(g x, F(x, y, z))\right] \\
+ & k_{4}\left[q_{2}(g x, F(y, z, x))+q_{2}(g y, F(z, x, y))+q_{2}(g z, F(x, y, z))\right] \\
+ & k_{5}\left[q_{2}(g y, F(x, y, z))+q_{2}(g z, F(y, z, x))+q_{2}(g x, F(z, x, y))\right] \\
= & k_{1}\left[q_{2}(g x, g y)+q_{2}(g y, g z)+q_{2}(g z, g x)\right] \\
+ & k_{2}\left[q_{2}(g x, g x)+q_{2}(g y, g y)+q_{2}(g z, g z)\right] \\
+ & k_{3}\left[q_{2}(g y, g y)+q_{2}(g z, g z)+q_{2}(g x, g x)\right] \\
+ & k_{4}\left[q_{2}(g x, g y)+q_{2}(g y, g z)+q_{2}(g z, g x)\right] \\
+ & k_{5}\left[q_{2}(g y, g x)+q_{2}(g z, g y)+q_{2}(g x, g z)\right] \\
\leq & k_{1}\left[q_{1}(g x, g y)+q_{1}(g y, g z)+q_{1}(g z, g x)\right] \\
+ & k_{2}\left[q_{1}(g x, g x)+q_{1}(g y, g y)+q_{1}(g z, g z)\right] \\
+ & k_{3}\left[q_{1}(g y, g y)+q_{1}(g z, g z)+q_{1}(g x, g x)\right] \\
+ & k_{4}\left[q_{1}(g x, g y)+q_{1}(g y, g z)+q_{1}(g z, g x)\right] \\
+ & k_{5}\left[q_{1}(g y, g x)+q_{1}(g z, g y)+q_{1}(g x, g z)\right] \\
= & \left(k_{1}+k_{4}\right)\left[q_{1}(g x, g y)+q_{1}(g y, g z)+q_{1}(g z, g x)\right] \\
+ & k_{5}\left[q_{1}(g y, g x)+q_{1}(g z, g y)+q_{1}(g x, g z)\right] .
\end{aligned}
$$

This implies that

$$
\begin{aligned}
& q_{1}(g x, g y)+q_{1}(g y, g z)+q_{1}(g z, g x) \\
& \quad \leq \frac{k_{5}}{1-k_{1}-k_{4}} \cdot\left[q_{1}(g y, g x)+q_{1}(g z, g y)+q_{1}(g x, g z)\right] .
\end{aligned}
$$

By similar arguments as above, we can show that

$$
\begin{aligned}
& q_{1}(g y, g x)+q_{1}(g z, g y)+q_{1}(g x, g z) \\
& \quad \leq \frac{k_{5}}{1-k_{1}-k_{4}} \cdot\left[q_{1}(g x, g y)+q_{1}(g y, g z)+q_{1}(g z, g x)\right] .
\end{aligned}
$$

Substituting (2.23) into (2.22), we have

$$
\begin{aligned}
& q_{1}(g x, g y)+q_{1}(g y, g z)+q_{1}(g z, g x) \\
& \quad \leq\left(\frac{k_{5}}{1-k_{1}-k_{4}}\right)^{2} \cdot\left[q_{1}(g x, g y)+q_{1}(g y, g z)+q_{1}(g z, g x)\right] .
\end{aligned}
$$

Since $\frac{k_{5}}{1-k_{1}-k_{4}}<1$, from (2.24), we must have $q_{1}(g x, g y)=q_{1}(g y, g z)=q_{1}(g z, g x)=0$. By Lemma 1.1, we get $g x=g y=g z$.

Finally, assume that $F$ and $g$ are $w$-compatible. Let $u=g x$, then we have $u=g x=$ $F(x, y, z)=g y=F(y, z, x)=g z=F(z, x, y)$, and so that

$$
g u=g g x=g(F(x, y, z))=F(g x, g y, g z)=F(u, u, u) .
$$


Consequently, $(u, u, u)$ is a tripled coincidence point of $F$ and $g$, and so $(g u, g u, g u)$ is a tripled point of coincidence of $F$ and $g$, and by its uniqueness, we get $g u=g x$. Thus, we obtain $F(u, u, u)=g u=u$. Therefore, $(u, u, u)$ is the unique common tripled fixed point of $F$ and $g$. This completes the proof of Theorem 2.1.

Remark 2.1 Theorem 2.1 improves and extends Theorem 2.1 of Shatanawi and Pitea [42] in the following aspects:

(1) The single quasi-partial metric extends to two quasi-partial metrics.

(2) The coupled fixed point extends to a tripled fixed point.

(3) The contractive condition defined by (1.1) is replaced by the new contractive condition defined by (2.2).

In Theorem 2.1, if we take $q_{1}(x, y)=q_{2}(x, y)$ for all $x, y \in X$, then we get the following.

Corollary 2.1 Let $(X, q)$ be a quasi-partial metric space, $F: X^{3} \rightarrow X$ and $g: X \rightarrow X$ be two mappings. Suppose that there exist $k_{1}, k_{2}, k_{3}, k_{4}$, and $k_{5}$ in $[0,1)$ with $k_{1}+k_{2}+k_{3}+2 k_{4}+k_{5}<1$ such that the condition

$$
\begin{aligned}
& q(F(x, y, z), F(u, v, w))+q(F(y, z, x), F(v, w, u))+q(F(z, x, y), F(w, u, v)) \\
& \leq k_{1}[q(g x, g u)+q(g y, g v)+q(z, w)] \\
&+k_{2}[q(g x, F(x, y, z))+q(g y, F(y, z, x))+q(g z, F(z, x, y))] \\
&+k_{3}[q(g u, F(u, v, w))+q(g v, F(v, w, u))+q(g w, F(w, u, v))] \\
&+k_{4}[q(g x, F(u, v, w))+q(g y, F(v, w, u))+q(g z, F(w, u, v))] \\
&+k_{5}[q(g u, F(x, y, z))+q(g v, F(y, z, x))+q(g w, F(z, x, y))]
\end{aligned}
$$

holds for all $x, y, z, u, v, w \in X$. Also, suppose we have the following hypotheses:

(i) $F\left(X^{3}\right) \subset g(X)$.

(ii) $g(X)$ is a complete subspace of $X$.

Then the mappings $F$ and $g$ have a tripled coincidence point $(x, y, z)$ satisfying

$$
g x=F(x, y, z)=g y=F(y, z, x)=F(z, x, y)=g z .
$$

Moreover, if $F$ and $g$ are $w$-compatible, then $F$ and $g$ have a unique common tripled fixed point of the form $(u, u, u)$.

Remark 2.2 Corollary 2.1 improves and extends Corollary 2.2 of Aydi and Abbas [41] to quasi-partial metric spaces.

Corollary 2.2 Let $q_{1}$ and $q_{2}$ be two quasi-metrics on $X$ such that $q_{2}(x, y) \leq q_{1}(x, y)$, for all $x, y \in X$, and $F: X^{3} \rightarrow X, g: X \rightarrow X$ be two mappings. Suppose that there exist $a_{i} \in[0,1)$ $(i=1,2,3, \ldots, 15)$ with

$$
\left(\sum_{i=1}^{9} a_{i}\right)+2\left(\sum_{i=10}^{12} a_{i}\right)+\left(\sum_{i=13}^{15} a_{i}\right)<1
$$


such that the condition

$$
\begin{aligned}
& q_{1}(F(x, y, z), F(u, v, w)) \\
& \leq a_{1} q_{2}(g x, g u)+a_{2} q_{2}(g y, g v)+a_{3} q_{2}(g z, g w) \\
&+a_{4} q_{2}(g x, F(x, y, z))+a_{5} q_{2}(g y, F(y, z, x))+a_{6} q_{2}(g z, F(z, x, y)) \\
&+a_{7} q_{2}(g u, F(u, v, w))+a_{8} q_{2}(g v, F(v, w, u))+a_{9} q_{2}(g w, F(w, u, v)) \\
&+a_{10} q_{2}(g x, F(u, v, w))+a_{11} q_{2}(g y, F(v, w, u))+a_{12} q_{2}(g z, F(w, u, v)) \\
&+a_{13} q_{2}(g u, F(x, y, z))+a_{14} q_{2}(g v, F(y, z, x))+a_{15} q_{2}(g w, F(z, x, y))
\end{aligned}
$$

holds for all $x, y, z, u, v, w \in X$. Also, suppose we have the following hypotheses:

(i) $F\left(X^{3}\right) \subset g(X)$.

(ii) $g(X)$ is a complete subspace of $X$ with respect to the quasi-partial metric $q_{1}$.

Then the mappings $F$ and $g$ have a tripled coincidence point $(x, y, z)$ satisfying

$$
g x=F(x, y, z)=g y=F(y, z, x)=g z=F(z, x, y) .
$$

Moreover, if $F$ and $g$ are $w$-compatible, then $F$ and $g$ have a unique common tripled fixed point of the form $(u, u, u)$.

Proof Given $x, y, z, u, v, w \in X$. It follows from (2.29) that

$$
\begin{aligned}
& q_{1}(F(x, y, z), F(u, v, w)) \\
& \leq a_{1} q_{2}(g x, g u)+a_{2} q_{2}(g y, g v)+a_{3} q_{2}(g z, g w) \\
&+a_{4} q_{2}(g x, F(x, y, z))+a_{5} q_{2}(g y, F(y, z, x))+a_{6} q_{2}(g z, F(z, x, y)) \\
&+a_{7} q_{2}(g u, F(u, v, w))+a_{8} q_{2}(g v, F(v, w, u))+a_{9} q_{2}(g w, F(w, u, v)) \\
&+a_{10} q_{2}(g x, F(u, v, w))+a_{11} q_{2}(g y, F(v, w, u))+a_{12} q_{2}(g z, F(w, u, v)) \\
&+a_{13} q_{2}(g u, F(x, y, z))+a_{14} q_{2}(g v, F(y, z, x))+a_{15} q_{2}(g w, F(z, x, y)), \\
& q_{1}(F(y, z, x), F(v, w, u)) \\
& \leq \\
& a_{1} q_{2}(g y, g v)+a_{2} q_{2}(g z, g w)+a_{3} q_{2}(g x, g u) \\
&+a_{4} q_{2}(g y, F(y, z, x))+a_{5} q_{2}(g z, F(z, x, y))+a_{6} q_{2}(g x, F(x, y, z)) \\
&+a_{7} q_{2}(g v, F(v, w, u))+a_{8} q_{2}(g w, F(w, u, v))+a_{9} q_{2}(g u, F(u, v, w)) \\
&+ a_{10} q_{2}(g y, F(v, w, u))+a_{11} q_{2}(g z, F(w, u, v))+a_{12} q_{2}(g x, F(u, v, w)) \\
&+a_{13} q_{2}(g v, F(y, z, x))+a_{14} q_{2}(g w, F(z, x, y))+a_{15} q_{2}(g u, F(x, y, z)),
\end{aligned}
$$

and

$$
\begin{aligned}
& q_{1}(F(z, x, y), F(w, u, v)) \\
& \quad \leq a_{1} q_{2}(g z, g w)+a_{2} q_{2}(g x, g u)+a_{3} q_{2}(g y, g v)
\end{aligned}
$$




$$
\begin{aligned}
& +a_{4} q_{2}(g z, F(z, x, y))+a_{5} q_{2}(g x, F(x, y, z))+a_{6} q_{2}(g y, F(y, z, x)) \\
& +a_{7} q_{2}(g w, F(w, u, v))+a_{8} q_{2}(g u, F(u, v, w))+a_{9} q_{2}(g v, F(v, w, u)) \\
& +a_{10} q_{2}(g z, F(w, u, v))+a_{11} q_{2}(g x, F(u, v, w))+a_{12} q_{2}(g y, F(v, w, u)) \\
& +a_{13} q_{2}(g w, F(z, x, y))+a_{14} q_{2}(g u, F(x, y, z))+a_{15} q_{2}(g v, F(y, z, x))
\end{aligned}
$$

Adding inequality (2.29) and (2.30) to inequality (2.31), we get

$$
\begin{aligned}
q_{1}\left(q_{1}(\right. & F(x, y, z), F(u, v, w))+F(y, z, x), F(v, w, u))+q_{1}(F(z, x, y), F(w, u, v)) \\
\leq & \left(a_{1}+a_{2}+a_{3}\right)\left[q_{2}(g x, g u)+q_{2}(g y, g v)+q_{2}(g z, g w)\right] \\
& +\left(a_{4}+a_{5}+a_{6}\right)\left[q_{2}(g x, F(x, y, z))+q_{2}(g y, F(y, z, x))+q_{2}(g z, F(z, x, y))\right] \\
& +\left(a_{7}+a_{8}+a_{9}\right)\left[q_{2}(g u, F(u, v, w))+q_{2}(g v, F(v, w, u))+q_{2}(g w, F(w, u, v))\right] \\
& +\left(a_{10}+a_{11}+a_{12}\right)\left[q_{2}(g x, F(u, v, w))+q_{2}(g y, F(v, w, u))+q_{2}(g z, F(w, u, v))\right] \\
& +\left(a_{13}+a_{14}+a_{15}\right)\left[q_{2}(g u, F(x, y, z))+q_{2}(g v, F(y, z, x))+q_{2}(g w, F(z, x, y))\right] .
\end{aligned}
$$

Therefore, the result follows from Theorem 2.1.

Remark 2.3 If we take $q_{1}(x, y)=q_{2}(x, y)=p(x, y)$ for all $x, y \in X$, where $p$ is a partial metric on $X$. Then Corollary 2.2 is reduced to Theorems 2.1 and 2.4 of Aydi and Abbas [41]. Corollary 2.2 also improves and extends Corollary 2.1 of Shatanawi and Pitea [35].

Corollary 2.3 Let $q_{1}$ and $q_{2}$ be two quasi-metrics on $X$ such that $q_{2}(x, y) \leq q_{1}(x, y)$, for all $x, y \in X$, and $F: X^{3} \rightarrow X, g: X \rightarrow X$ be two mappings. Suppose that there exists $k \in[0,1)$ such that the condition

$$
\begin{aligned}
& q_{1}(F(x, y, z), F(u, v, w))+q_{1}(F(y, z, x), F(v, w, u))+q_{1}(F(z, x, y), F(w, u, v)) \\
& \quad \leq k\left[q_{2}(g x, g u)+q_{2}(g y, g v)+q_{2}(g z, g w)\right]
\end{aligned}
$$

holds for all $x, y, z, u, v, w \in X$. Also, suppose we have the following hypotheses:

(i) $F\left(X^{3}\right) \subset g(X)$.

(ii) $g(X)$ is a complete subspace of $X$ with respect to the quasi-partial metric $q_{1}$.

Then the mappings $F$ and $g$ have a tripled coincidence point $(x, y, z)$ satisfying

$$
g x=F(x, y, z)=g y=F(y, z, x)=g z=F(z, x, y) .
$$

Moreover, if $F$ and $g$ are $w$-compatible, then $F$ and $g$ have a unique common tripled fixed point of the form $(u, u, u)$.

Corollary 2.4 Let $q_{1}$ and $q_{2}$ be two quasi-metrics on $X$ such that $q_{2}(x, y) \leq q_{1}(x, y)$, for all $x, y \in X$, and $F: X^{3} \rightarrow X, g: X \rightarrow X$ be two mappings. Suppose that there exists $k \in[0,1)$ such that the condition

$$
\begin{aligned}
& q_{1}(F(x, y, z), F(u, v, w))+q_{1}(F(y, z, x), F(v, w, u))+q_{1}(F(z, x, y), F(w, u, v)) \\
& \quad \leq k\left[q_{2}(g x, F(x, y, z))+q_{2}(g y, F(y, z, x))+q_{2}(F(g z, F(z, x, y)))\right]
\end{aligned}
$$


holds for all $x, y, z, u, v, w \in X$. Also, suppose we have the following hypotheses:

(i) $F\left(X^{3}\right) \subset g(X)$.

(ii) $g(X)$ is a complete subspace of $X$ with respect to the quasi-partial metric $q_{1}$.

Then the mappings $F$ and $g$ have a tripled coincidence point $(x, y, z)$ satisfying

$$
g x=F(x, y, z)=g y=F(y, z, x)=g z=F(z, x, y) .
$$

Moreover, if $F$ and $g$ are $w$-compatible, then $F$ and $g$ have a unique common tripled fixed point of the form $(u, u, u)$.

Corollary 2.5 Let $q_{1}$ and $q_{2}$ be two quasi-metrics on $X$ such that $q_{2}(x, y) \leq q_{1}(x, y)$, for all $x, y \in X$, and $F: X^{3} \rightarrow X, g: X \rightarrow X$ be two mappings. Suppose that there exists $k \in[0,1)$ such that the condition

$$
\begin{aligned}
& q_{1}(F(x, y, z), F(u, v \cdot w))+q_{1}(F(y, z, x), F(v, w, u))+q_{1}(F(z, x, y), F(w, u, v)) \\
& \leq k\left[q_{2}(g u, F(u, v, w))+q_{2}(g v, F(v, w, u))+q_{2}(g w, F(w, u, v))\right]
\end{aligned}
$$

holds for all $x, y, z, u, v, w \in X$. Also, suppose we have the following hypotheses:

(i) $F\left(X^{3}\right) \subset g(X)$.

(ii) $g(X)$ is a complete subspace of $X$ with respect to the quasi-partial metric $q_{1}$.

Then the mappings $F$ and $g$ have a tripled coincidence point $(x, y, z)$ satisfying

$$
g x=F(x, y, z)=g y=F(y, z, x)=g z=F(z, x, y) .
$$

Moreover, if $F$ and $g$ are $w$-compatible, then $F$ and $g$ have a unique common tripled fixed point of the form $(u, u, u)$.

Remark 2.4 Corollaries 2.3-2.5 improve and extend Corollaries 2.2-2.4 of Shatanawi and Pitea [42] in the following aspects:

(1) The single quasi-partial metric extends to two quasi-partial metrics.

(2) The coupled fixed point extends to a tripled fixed point.

Corollary 2.6 Let $q_{1}$ and $q_{2}$ be two quasi-metrics on $X$ such that $q_{2}(x, y) \leq q_{1}(x, y)$, for all $x, y \in X$, and $F: X^{3} \rightarrow X, g: X \rightarrow X$ be two mappings. Suppose that there exists $k \in\left[0, \frac{1}{2}\right)$ such that the condition

$$
\begin{aligned}
& q_{1}(F(x, y, z), F(u, v, w))+q(F(y, z, x), F(v, w, u))+q_{1}(F(z, x, y), F(w, u, v)) \\
& \quad \leq k\left[q_{2}(g x, F(u, v, w))+q_{2}(g y, F(v, w, u))+q_{2}(g z, F(w, u, v))\right]
\end{aligned}
$$

holds for all $x, y, z, u, v, w \in X$. Also, suppose we have the following hypotheses: 
(i) $F\left(X^{3}\right) \subset g(X)$.

(ii) $g(X)$ is a complete subspace of $X$ with respect to the quasi-partial metric $q_{1}$.

Then the mappings $F$ and $g$ have a tripled coincidence point $(x, y, z)$ satisfying

$$
g x=F(x, y, z)=g y=F(y, z, x)=g z=F(z, x, y) .
$$

Moreover, if $F$ and $g$ are $w$-compatible, then $F$ and $g$ have a unique common tripled fixed point of the form $(u, u, u)$.

Corollary 2.7 Let $q_{1}$ and $q_{2}$ be two quasi-metrics on $X$ such that $q_{2}(x, y) \leq q_{1}(x, y)$, for all $x, y \in X$, and $F: X^{3} \rightarrow X, g: X \rightarrow X$ be two mappings. Suppose that there exists $k \in[0,1)$ such that the condition

$$
\begin{aligned}
& q_{1}(F(x, y, z), F(u, v, w))+q(F(y, z, x), F(v, w, u))+q_{1}(F(z, x, y), F(w, u, v)) \\
& \quad \leq k\left[q_{2}(g u, F(x, y, z))+q_{2}(g v, F(y, z, x))+q_{2}(g w, F(z, x, y))\right]
\end{aligned}
$$

holds for all $x, y, z, u, v, w \in X$. Also, suppose we have the following hypotheses:

(i) $F\left(X^{3}\right) \subset g(X)$.

(ii) $g(X)$ is a complete subspace of $X$ with respect to the quasi-partial metric $q_{1}$.

Then the mappings $F$ and $g$ have a tripled coincidence point $(x, y, z)$ satisfying

$$
g x=F(x, y, z)=g y=F(y, z, x)=g z=F(z, x, y) .
$$

Moreover, if $F$ and $g$ are $w$-compatible, then $F$ and $g$ have a unique common tripled fixed point of the form $(u, u, u)$.

Let $g=I_{X}$ (the identity mapping) in Theorem 2.1 and Corollaries 2.1-2.7. Then we have the following results.

Corollary 2.8 Let $q_{1}$ and $q_{2}$ be two quasi-metrics on $X$ such that $q_{2}(x, y) \leq q_{1}(x, y)$, for all $x, y \in X$, and $F: X^{3} \rightarrow X$ be a mapping. Suppose that there exist $k_{1}, k_{2}, k_{3}, k_{4}$, and $k_{5}$ in $[0,1)$ with $k_{1}+k_{2}+k_{3}+2 k_{4}+k_{5}<1$ such that the condition

$$
\begin{aligned}
& q_{1}(F(x, y, z), F(u, v, w))+q_{1}(F(y, z, x), F(v, w, u))+q_{1}(F(z, x, y), F(w, u, v)) \\
& \leq k_{1}\left[q_{2}(x, u)+q_{2}(y, v)+q_{2}(z, w)\right] \\
&+k_{2}\left[q_{2}(x, F(x, y, z))+q_{2}(y, F(y, z, x))+q_{2}(z, F(z, x, y))\right] \\
&+k_{3}\left[q_{2}(u, F(u, v, w))+q_{2}(v, F(v, w, u))+q_{2}(w, F(w, u, v))\right] \\
&+k_{4}\left[q_{2}(x, F(u, v, w))+q_{2}(y, F(v, w, u))+q_{1}(z, F(w, u, v))\right] \\
&+k_{5}\left[q_{2}(u, F(x, y, z))+q_{2}(v, F(y, z, x))+q_{2}(w, F(z, x, y))\right]
\end{aligned}
$$

holds for all $x, y, z, u, v, w \in X$. If $\left(X, q_{1}\right)$ is a complete quasi-partial metric space, then the mapping $F$ has a unique tripled fixed point of the form $(u, u, u)$. 
Corollary 2.9 Let $(X, q)$ be a complete quasi-partial metric space, $F: X^{3} \rightarrow X$ be a mapping. Suppose that there exist $k_{1}, k_{2}, k_{3}, k_{4}$, and $k_{5}$ in $[0,1)$ with $k_{1}+k_{2}+k_{3}+2 k_{4}+k_{5}<1$ such that the condition

$$
\begin{aligned}
q(F( & x, y, z), F(u, v, w))+q(F(y, z, x), F(v, w, u))+q(F(z, x, y), F(w, u, v)) \\
\leq & k_{1}[q(x, u)+q(y, v)+q(z, w)] \\
& +k_{2}[q(x, F(x, y, z))+q(y, F(y, z, x))+q(z, F(z, x, y))] \\
& +k_{3}[q(u, F(u, v, w))+q(v, F(v, w, u))+q(w, F(w, u, v))] \\
& +k_{4}[q(x, F(u, v, w))+q(y, F(v, w, u))+q(z, F(w, u, v))] \\
& +k_{5}[q(u, F(x, y, z))+q(v, F(y, z, x))+q(w, F(z, x, y))]
\end{aligned}
$$

holds for all $x, y, z, u, v, w \in X$. Then $F$ has a unique tripled fixed point of the form $(u, u, u)$.

Remark 2.5 Corollary 2.9 improves and extends Corollary 2.5 of Shatanawi and Pitea [42], the contractive condition is replaced by the new contractive condition defined by (2.39).

Corollary 2.10 Let $(X, p)$ be a complete partial metric space, $F: X^{3} \rightarrow X$ be a mapping. Suppose that there exist $k_{1}, k_{2}, k_{3}, k_{4}$, and $k_{5}$ in $[0,1)$ with $k_{1}+k_{2}+k_{3}+2 k_{4}+k_{5}<1$ such that the condition

$$
\begin{aligned}
& p(F(x, y, z), F(u, v, w)) \\
& \leq k_{1}[p(x, u)+p(y, v)+p(z, w)] \\
&+k_{2}[p(x, F(x, y, z))+p(y, F(y, z, x))+p(z, F(z, x, y))] \\
&+k_{3}[p(u, F(u, v, w))+p(v, F(v, w, u))+p(w, F(w, u, v))] \\
&+k_{4}[p(x, F(u, v, w))+p(y, F(v, w, u))+p(z, F(w, u, v))] \\
&+k_{5}[p(u, F(x, y, z))+p(v, F(y, z, x))+p(w, F(z, x, y))]
\end{aligned}
$$

holds for all $x, y, z, u, v, w \in X$. Then the mapping $F$ has a unique tripled fixed point of the form $(u, u, u)$.

Corollary 2.11 Let $q_{1}$ and $q_{2}$ be two quasi-metrics on $X$ such that $q_{2}(x, y) \leq q_{1}(x, y)$, for all $x, y \in X$, and $F: X^{3} \rightarrow X$ be a mapping. Suppose that there exist $a_{i} \in[0,1)(i=1,2,3, \ldots, 15)$ with

$$
\left(\sum_{i=1}^{9} a_{i}\right)+2\left(\sum_{i=10}^{12} a_{i}\right)+\left(\sum_{i=13}^{15} a_{i}\right)<1
$$

such that the condition

$$
\begin{aligned}
& q_{1}(F(x, y, z), F(u, v, w)) \\
& \quad \leq a_{1} q_{2}(x, u)+a_{2} q_{2}(y, v)+a_{3} q_{2}(z, w)
\end{aligned}
$$




$$
\begin{aligned}
& +a_{4} q_{2}(x, F(x, y, z))+a_{5} q_{2}(y, F(y, z, x))+a_{6} q_{2}(z, F(z, x, y)) \\
& +a_{7} q_{2}(u, F(u, v, w))+a_{8} q_{2}(v, F(v, w, u))+a_{9} q_{2}(w, F(w, u, v)) \\
& +a_{10} q_{2}(x, F(u, v, w))+a_{11} q_{2}(y, F(v, w, u))+a_{12} q_{2}(z, F(w, u, v)) \\
& +a_{13} q_{2}(u, F(x, y, z))+a_{14} q_{2}(v, F(y, z, x))+a_{15} q_{2}(w, F(z, x, y))
\end{aligned}
$$

holds for all $x, y, z, u, v, w \in X$. If $\left(X, q_{1}\right)$ is a complete quasi-partial metric space. Then the mapping $F$ has a unique coupled fixed point of the form $(u, u, u)$.

Remark 2.6 Corollary 2.11 improves and extends Corollary 2.6 of Shatanawi and Pitea [42] in the following aspects:

(1) The single quasi-partial metric extends to two quasi-partial metrics.

(2) The coupled fixed point extends to a tripled fixed point.

(3) The contractive condition is replaced by the new contractive condition defined by (2.42).

Corollary 2.12 Let $q_{1}$ and $q_{2}$ be two quasi-metrics on $X$ such that $q_{2}(x, y) \leq q_{1}(x, y)$, for all $x, y \in X$, and $F: X^{3} \rightarrow X$ be a mapping. Suppose that there exists $k \in[0,1)$ such that the condition

$$
\begin{aligned}
& q_{1}(F(x, y, z), F(u, v, w))+q_{1}(F(y, z, x), F(v, w, u))+q_{1}(F(z, x, y), F(w, u, v)) \\
& \quad \leq k\left[q_{2}(x, u)+q_{2}(y, v)+q_{2}(z, w)\right]
\end{aligned}
$$

holds for all $x, y, z, u, v, w \in X$. If $\left(X, q_{1}\right)$ is a complete quasi-partial metric space. Then the mapping $F$ has a unique tripled fixed point of the form $(u, u, u)$.

Corollary 2.13 Let $q_{1}$ and $q_{2}$ be two quasi-metrics on $X$ such that $q_{2}(x, y) \leq q_{1}(x, y)$, for all $x, y \in X$, and $F: X^{3} \rightarrow X$ be a mapping. Suppose that there exists $k \in[0,1)$ such that the condition

$$
\begin{aligned}
& q_{1}(F(x, y, z), F(u, v, w))+q_{1}(F(y, z, x), F(v, w, u))+q_{1}(F(z, x, y), F(w, u, v)) \\
& \quad \leq k\left[q_{2}(x, F(x, y, z))+q_{2}(y, F(y, z, x))+q_{2}(z, F(z, x, y))\right]
\end{aligned}
$$

holds for all $x, y, z, u, v, w \in X$. If $\left(X, q_{1}\right)$ is a complete quasi-partial metric space. Then the mapping $F$ has a unique tripled fixed point of the form $(u, u, u)$.

Corollary 2.14 Let $q_{1}$ and $q_{2}$ be two quasi-metrics on $X$ such that $q_{2}(x, y) \leq q_{1}(x, y)$, for all $x, y \in X$, and $F: X^{3} \rightarrow X$ be a mapping. Suppose that there exists $k \in[0,1)$ such that the condition

$$
\begin{aligned}
& q_{1}(F(x, y, z), F(u, v, w))+q(F(y, z, x), F(v, w, u))+q_{1}(F(z, x, y), F(w, u, v)) \\
& \quad \leq k\left[q_{2}(u, F(u, v, w))+q_{2}(v, F(v, w, u))+q_{2}(w, F(w, u, v))\right]
\end{aligned}
$$

holds for all $x, y, z, u, v, w \in X$. If $\left(X, q_{1}\right)$ is a complete quasi-partial metric space. Then the mapping $F$ has a unique tripled fixed point of the form $(u, u, u)$. 
Remark 2.7 Corollaries 2.12-2.14 improve and extend Corollaries 2.7-2.9 of Shatanawi and Pitea [42] in the following aspects:

(1) The single quasi-partial metric extends to two quasi-partial metrics.

(2) The coupled fixed point extends to a tripled fixed point.

Corollary 2.15 Let $q_{1}$ and $q_{2}$ be two quasi-metrics on $X$ such that $q_{2}(x, y) \leq q_{1}(x, y)$, for all $x, y \in X$, and $F: X^{3} \rightarrow X$ be a mapping. Suppose that there exists $k \in\left[0, \frac{1}{2}\right)$ such that the condition

$$
\begin{aligned}
& q_{1}(F(x, y, z), F(u, v, w))+q(F(y, z, x), F(v, w, u))+q_{1}(F(z, x, y), F(w, u, v)) \\
& \quad \leq k\left[q_{2}(x, F(u, v, w))+q_{2}(y, F(v, w, u))+q_{2}(z, F(w, u, v))\right]
\end{aligned}
$$

holds for all $x, y, z, u, v, w \in X$. If $\left(X, q_{1}\right)$ is a complete quasi-partial metric space. Then the mapping $F$ has a unique tripled fixed point of the form $(u, u, u)$.

Corollary 2.16 Let $q_{1}$ and $q_{2}$ be two quasi-metrics on $X$ such that $q_{2}(x, y) \leq q_{1}(x, y)$, for all $x, y \in X$, and $F: X^{3} \rightarrow X$ be a mapping. Suppose that there exists $k \in[0,1)$ such that the condition

$$
\begin{aligned}
& q_{1}(F(x, y, z), F(u, v, w))+q_{1}(F(y, z, x), F(v, w, u))+q_{1}(F(z, x, y), F(w, u, v)) \\
& \quad \leq k\left[q_{2}(u, F(x, y, z))+q_{2}(v, F(y, z, x))+q_{2}(w, F(z, x, y))\right]
\end{aligned}
$$

holds for all $x, y, z, u, v, w \in X$. If $\left(X, q_{1}\right)$ is a complete quasi-partial metric space. Then the mapping $F$ has a unique tripled fixed point of the form $(u, u, u)$.

Now, we introduce an example to support our results.

Example 2.1 Let $X=[0,1]$, and two quasi-partial metrics $q_{1}, q_{2}$ on $X$ be given as

$$
q_{1}(x, y)=|x-y|+x \quad \text { and } \quad q_{2}(x, y)=\frac{1}{2}[|x-y|+x]
$$

for all $x, y \in X$. Also, define $F: X^{3} \rightarrow X$ and $g: X \rightarrow X$ as

$$
F(x, y, z)=\frac{x+y+z}{27} \text { and } g x=\frac{x}{3}
$$

for all $x, y, z \in X$. Then

(1) $\left(X, q_{1}\right)$ is a complete quasi-partial metric space.

(2) $F\left(X^{3}\right) \subset X$.

(3) $F$ and $g$ are $w$-compatible.

(4) For any $x, y, z, u, v, w \in X$, we have

$$
\begin{aligned}
& q_{1}(F(x, y, z), F(u, v, w))+q_{1}(F(y, z, x)+F(v, w, u))+q_{1}(F(z, x, y), F(w, u, v)) \\
& \quad \leq \frac{1}{3}\left(q_{2}(g x, g u)+q_{2}(g y, g v)+q_{2}(g z, g w)\right) .
\end{aligned}
$$


Proof The proofs of (1), (2), and (3) are clear. Next we show that (4). In fact, for $x, y, z, u, v, w \in X$, we have

$$
\begin{aligned}
& q_{1}(F(x, y, z), F(u, v, w))+q_{1}(F(y, z, x)+F(v, w, u))+q_{1}(F(z, x, y), F(w, u, v)) \\
& \quad=q_{1}\left(\frac{x+y+z}{27}, \frac{u+v+w}{27}\right)+q_{1}\left(\frac{y+z+x}{27}, \frac{v+w+u}{27}\right)+q_{1}\left(\frac{z+x+y}{27}, \frac{w+u+v}{27}\right) \\
& \quad=\frac{1}{9}(|x+y+z-(u+v+w)|+(x+y+z)) \\
& \quad \leq \frac{1}{9}(|x-u|+|y-v|+|z-w|+x+y+z) \\
& \quad=\frac{1}{3}\left(\left|\frac{1}{3} x-\frac{1}{3} u\right|+\frac{1}{3} x+\left|\frac{1}{3} y-\frac{1}{3} v\right|+\frac{1}{3} y+\left|\frac{1}{3} z-\frac{1}{3} w\right|+\frac{1}{3} z\right) \\
& \quad=\frac{1}{3}\left(q_{2}(g x, g u)+q_{2}(g y, g v)+q_{2}(g z, g w)\right) .
\end{aligned}
$$

Thus, $F$ and $g$ satisfy all the hypotheses of Corollary 2.3. So, $F$ and $g$ have a unique common coupled fixed point. Here $(0,0,0)$ is the unique common tripled fixed point of $F$ and $g$.

\section{Competing interests}

The author declares that they have no competing interests.

\section{Acknowledgements}

The author is grateful to the reviewers for suggestions which improved the contents of the article. This work is supported by the National Natural Science Foundation of China (11271105, 11071169), the Natural Science Foundation of Zhejiang Province (Y61 10287, LY12A01030) and the Natural Science Foundation of Shandong Province (ZR2013AL015).

\section{Received: 7 January 2014 Accepted: 4 March 2014 Published: 20 Mar 2014}

\section{References}

1. Matthews, SG: Partial metric topology. In: General Topology and Its Applications, Proc. 8th Summer Conf., Queen's College, 1992. Annals of the New York Academy of Sciences, vol. 728, pp. 183-197. New York Academy of Science, New York (1994)

2. Abdeljawad, T, Karapinar, E, Taş, K: Existence and uniqueness of a common fixed point on partial metric spaces. Appl. Math. Lett. 24(11), 1900-1904 (2011)

3. Abdeljawad, T, Karapinar, E, Taş, K: A generalized contraction principle with control functions on partial metric spaces. Comput. Math. Appl. 63(3), 716-719 (2012)

4. Abdeljawad, T: Fixed points and generalized weakly contractive mappings in partial metric spaces. Math. Comput. Model. 54(11-12), 2923-2927 (2011)

5. Altun, I, Acar, Ö: Fixed point theorems for weak contractions in the sense of Berinde on partial metric spaces. Topol. Appl. 159, 2642-2648 (2012)

6. Altun, I, Erduran, A: Fixed point theorems for monotone mappings on partial metric spaces. Fixed Point Theory Appl. 2011, 508730 (2011). doi:10.1155/2011/508730

7. Altun, I, Simsek, H: Some fixed point theorems on dualistic partial metric spaces. J. Adv. Math. Stud. 1(1-2), 1-8 (2008)

8. Altun, I, Sola, F, Simsek, H: Generalized contractions on partial metric spaces. Topol. Appl. 157(18), $2778-2785$ (2010)

9. Altun, I, Sadarangani, K: Corrigendum to 'Generalized contractions on partial metric spaces' [Topology Appl. 157 (2010) 2778-2785]. Topol. Appl. 158(13), 1738-1740 (2011)

10. Amiri, P, Rezapour, S: Fixed point of multi-valued operators on partial metric spaces. Anal. Theory Appl. 29(2), 158-168 (2013). doi:10.4208/ata.2013.v29.n2.7

11. Aydi, H: Some fixed point results in ordered partial metric spaces. J. Nonlinear Sci. Appl. 4(2), 1-12 (2011)

12. Aydi, H: Some coupled fixed point results on partial metric spaces. Int. J. Math. Sci. 2011, 647091 (2011)

13. Aydi, H: Fixed point theorems for generalized weakly contractive in ordered partial metric spaces. J. Nonlinear Anal. Optim., Theory Appl. 2(2), 269-284 (2011)

14. Aydi, H, Karapinar, E, Shatanawi, W: Coupled fixed point results for $(\psi, \varphi)$-weakly contractive condition in ordered partial metric spaces. Comput. Math. Appl. 62, 4449-4460 (2011)

15. Bari, CD, Milojević, M, Radenović, S, Vetro, P: Common fixed points for self-mappings on partial metric spaces. Fixed Point Theory Appl. 2012, 140 (2012). doi:10.1186/1687-1812-2012-140

16. Chakkrid, K: Modified proof of Caristi's fixed point theorem on partial metric spaces. J. Inequal. Appl. 2013,210 (2013). doi:10.1186/1029-242X-2013-210

17. Chen, C, Zhu, C: Fixed point theorems for weakly C-contractive mappings in partial metric spaces. Fixed Point Theory Appl. 2013, 107 (2013). doi:10.1186/1687-1812-2013-107 
18. Ćirić, L, Samet, B, Aydi, H, Vetro, C: Common fixed point results of generalized contractions on partial metric spaces and application. Appl. Math. Comput. 218, 2398-2406 (2011)

19. Golubović, Z, Kadelburg, Z, Radenović, S: Coupled coincidence points of mappings in ordered partial metric spaces. Abstr. Appl. Anal. 2012, 192581 (2012). doi:10.1155/2012/192581

20. Karapinar, E, Erhan, I: Fixed point theorems for operators on partial metric spaces. Appl. Math. Lett. 24, 1894-1899 (2011)

21. Nashine, HK, Kadelburg, Z, Radenović, S: Common fixed point theorems for weakly isotone increasing mappings in ordered partial metric spaces. Math. Comput. Model. 57, 2355-2365 (2013)

22. Oltra, S, Valero, O: Banach's fixed point theorem for partial metric spaces. Rend. Ist. Mat. Univ. Trieste 36(1-2), 17-26 (2004)

23. Romaguera, S: A Kirk type characterization of completeness for partial metric spaces. Fixed Point Theory Appl. 2010 493298 (2010). doi:10.1155/2010/493298

24. Romaguera, S: Fixed point theorems for generalized contractions on partial metric spaces. Topol. Appl. 159, 194-199 (2010)

25. Samet, B, Rajović, M, Lazović, R, Stoijković, R: Common fixed point results for nonlinear contractions in ordered partial metric spaces. Fixed Point Theory Appl. 2011, 71 (2011). doi:10.1186/1687-1812-2011-71

26. Shatanawi, W, Nashine, HK: A generalization of Banach's contraction principle of nonlinear contraction in a partial metric spaces. J. Nonlinear Sci. Appl. 5, 37-43 (2012)

27. Shatanawi, W, Nashine, HK, Tahat, N: Generalization of some coupled fixed point results on partial metric spaces. Int. J. Math. Math. Sci. 2012, 686801 (2012)

28. Shatanawi, W, Samet, B, Abbas, M: Coupled fixed point theorems for mixed monotone mappings in ordered partial metric spaces. Math. Comput. Model. 55, 680-687 (2012)

29. Shatanawi, W, Postolache, M: Coincidence and fixed point results for generalized weak contractions in the sense of Berinde on partial metric spaces. Fixed Point Theory Appl. 2013, 54 (2013). doi:10.1186/1687-1812-2013-54

30. Radenović, S: Remarks on some coupled fixed point results in partial metric spaces. Nonlinear Funct. Anal. Appl. 18(1), 39-50 (2013)

31. Nashine, HK, Kadelburg, Z, Radenović, S: Fixed point theorems via various cyclic contractive conditions in partial metric spaces. Publ. Inst. Math. (Beograd) (N.S.) 93(107), 69-93 (2013)

32. Valero, O: On Banach fixed point theorems for partial metric spaces. Appl. Gen. Topol. 6(2), $229-240$ (2005)

33. Haghi, RH, Rezapour, Sh, Shahzad, N: Be careful on partial metric fixed point results. Topol. Appl. 160, 450-454 (2013)

34. Karapinar, E, Erhan, I, Öztürk, A: Fixed point theorems on quasi-partial metric spaces. Math. Comput. Model. 57, 2442-2448 (2013). doi:10.1016/j.mcm.2012.06.036

35. Bhaskar, TG, Lakshmikantham, V: Fixed point theorems in partially ordered metric spaces and applications. Nonlinear Anal. 65, 1379-1393 (2006)

36. Lakshmikantham, V, Ćirić, L: Coupled fixed point theorems for nonlinear contractions in partially ordered metric spaces. Nonlinear Anal. 70, 4341-4349 (2009)

37. Abbas, M, Khan, MA, Radenović, S: Common coupled fixed point theorem in cone metric space for $w$-compatible mappings. Appl. Math. Comput. 217, 195-202 (2010). doi:10.1016/j.amc.2010.05.042

38. Samet, B, Vetro, C: Coupled fixed point, $f$-invariant set and fixed point of N-order. Ann. Funct. Anal. 1(2), 46-56 (2010)

39. Berinde, V, Borcut, M: Tripled fixed point theorems for contractive type mappings in partially ordered metric spaces. Nonlinear Anal. 74(15), 4889-4897 (2011)

40. Aydi, H, Abbas, $M$, Sintunavarat, $W$, Kumam, P: Tripled fixed point of $W$-compatible mappings in abstract metric spaces. Fixed Point Theory Appl. 2012, 134 (2012). doi:10.1186/1687-1812-2012-134

41. Aydi, H, Abbas, M: Tripled coincidence and fixed point results in partial metric spaces. Appl. Gen. Topol. 13(2), 193-206 (2012)

42. Shatanawi, W, Pitea, A: Some coupled fixed point theorems in quasi-partial metric spaces. Fixed Point Theory Appl. 2013, 153 (2013). doi:10.1186/1687-1812-2013-153

43. Abbas, M, Khan, AR, Nazir, T: Coupled common fixed point results in two generalized metric spaces. Appl. Math. Comput. 217, 6328-6336 (2011). doi:10.1016/j.amc.2011.01.006

44. Aydi, H, Karapinar, E, Postolache, M: Tripled coincidence point theorems for weak $\phi$-contractions in partially ordered metric spaces. Fixed Point Theory Appl. 2012, 44 (2012)

45. Abbas, M, Nazir, T, Radenović, S: Common fixed point of generalized weakly contractive maps in partially ordered G-metric spaces. Appl. Math. Comput. 218(18), 9383-9395 (2012)

46. Aydi, H, Postolache, M, Shatanawi, W: Coupled fixed point results for $(\psi, \phi)$-weakly contractive mappings in ordered G-metric spaces. Comput. Math. Appl. 63(1), 298-309 (2012)

47. Abbas, M, Sintunavarat, W, Kumam, P: Coupled fixed point of generalized contractive mappings on partially ordered G-metric spaces. Fixed Point Theory Appl. 2012, 31 (2012). doi:10.1186/1687-1812-2012-31

48. Aydi, H, Damjanović, B, Samet, B, Shatanawi, W: Coupled fixed point theorems for nonlinear contractions in partially ordered G-metric spaces. Math. Comput. Model. 54(9-10), 2443-2450 (2011)

49. Berinde, V: Generalized coupled fixed point theorems for mixed monotone mappings in partially ordered metric spaces. Nonlinear Anal. 74(18), 7347-7355 (2011)

50. Berinde, $\mathrm{V}$ : Coupled coincidence point theorems for mixed monotone nonlinear operators. Comput. Math. Appl. 64(6), 1770-1777 (2012)

51. Berinde, $\mathrm{V}$ : Coupled fixed point theorems for $\varphi$-contractive mixed monotone mappings in partially ordered metric spaces. Nonlinear Anal. 75(6), 3218-3228 (2012)

52. Berinde, V, Păcurar, M: Coupled fixed point theorems for generalized symmetric Meir-Keeler contractions in ordered metric spaces. Fixed Point Theory Appl. 2012, 115 (2012)

53. Chandok, S, Mustafa, Z, Postolache, M: Coupled common fixed point theorems for mixed $g$-monotone mappings in partially ordered G-metric spaces. Sci. Bull. "Politeh." Univ. Buchar., Ser. A, Appl. Math. Phys. 75(4), 13-26 (2013)

54. Cho, YJ, Rhoades, BE, Saadati, R, Samet, B, Shatanawi, W: Nonlinear coupled fixed point theorems in ordered generalized metric spaces with integral type. Fixed Point Theory Appl. 2012, 8 (2012). doi:10.1186/1687-1812-2012-8

55. Choudhury, BS, Metiya, N, Postolache, M: A generalized weak contraction principle with applications to coupled coincidence point problems. Fixed Point Theory Appl. 2013, 152 (2013). doi:10.1186/1687-1812-2013-152 
56. Gu, F, Wang, L: Some coupled fixed-point theorems in two quasi-partial metric spaces. Fixed Point Theory Appl. 2014 19 (2014)

57. Gu, F, Yin, Y: A new common coupled fixed point theorem in generalized metric space and applications to integral equations. Fixed Point Theory Appl. 2013, 266 (2013). doi:10.1186/1687-1812-2013-266

58. Gu, F, Zhou, S: Coupled common fixed point theorems for a pair of commuting mappings in partially ordered G-metric spaces. Fixed Point Theory Appl. 2013, 64 (2013). doi:10.1186/1687-1812-2013-64

59. Karapinar, E: Coupled fixed point theorems for nonlinear contractions in cone metric spaces. Comput. Math. Appl. 59, 3656-3668 (2010)

60. Olatinwo, MO, Postolache, M: Stability results for Jungck-type iterative processes in convex metric spaces. Appl. Math Comput. 218(12), 6727-6732 (2012)

61. Qiu, Z, Hong, S: Coupled fixed points for multivalued mappings in fuzzy metric spaces. Fixed Point Theory Appl. 2013 162 (2013). doi:10.1186/1687-1812-2013-162

62. Samet, B: Coupled fixed point theorems for a generalized Meir-Keeler contraction in partially ordered metric spaces. Nonlinear Anal. 72, 4508-4517 (2010)

63. Shatanawi, W: On $w$-compatible mappings and common coupled coincidence point in cone metric spaces. Appl. Math. Lett. 25, 925-931 (2012)

64. Shatanawi, W, Abbas, M, Nazir, T: Common coupled coincidence and coupled fixed point results in two generalized metric spaces. Fixed Point Theory Appl. 2011, 80 (2011). doi:10.1186/1687-1812-2011-80

65. Shatanawi, W, Pitea, A: Omega-distance and coupled fixed point in G-metrics spaces. Fixed Point Theory Appl. 2013, 208 (2013)

66. Shatanawi, W, Pitea, A: Fixed and coupled fixed point theorems of omega-distance for nonlinear contraction. Fixed Point Theory Appl. 2013, 275 (2013)

67. Shatanawi, W, Postolache, M: Common fixed point results for mappings under nonlinear contraction of cyclic form in ordered metric spaces. Fixed Point Theory Appl. 2013, 60 (2013)

68. Shatanawi, W, Postolache, M: Common fixed point theorems for dominating and weak annihilator mappings in ordered metric spaces. Fixed Point Theory Appl. 2013, 271 (2013)

10.1186/1687-1812-2014-71

Cite this article as: Gu: Some common tripled fixed point results in two quasi-partial metric spaces. Fixed Point Theory and Applications 2014, 2014:71

\section{Submit your manuscript to a SpringerOpen ${ }^{\circ}$ journal and benefit from:}

- Convenient online submission

- Rigorous peer review

- Immediate publication on acceptance

- Open access: articles freely available online

- High visibility within the field

- Retaining the copyright to your article 